\title{
MONTE CARLO CALCULATIONS OF A BISMUTH GERMANATE SCINTILLATION DETECTOR
}

\author{
Salih M. ALKHAFAJI * \\ Department of Nuclear Engineering, The University of Michigan, Ann Arbor, Michigan 48109, U.S.A.
}

Received 16 June 1980 and in revised form 13 February 1981

Intrinsic efficiency, radial distribution of deposited energy and other properties of importance were investigated for bismuth germanate slabs via Monte Carlo calculation. The calculations were done for different slab thicknesses in the range $1 / 8^{\prime \prime}-2^{\prime \prime}$, and over the energy range 140-999 $\mathrm{keV}$. An examination of the data shows the possible advantage of the use of BGO as scintillation detector for high energy gamma ray and positron imaging. The data do not show very strong dependence of the image resolution on the thickness of the slab.

For the last decade, most of the emphasis in nuclear medicine has been placed on radioisotopes which emit gamma rays of energy $\sim 200-400 \mathrm{keV}$. The reasons for this relate to the unique problems encountered in imaging a spatial distribution of radioactivity. There are two basic problems related to imaging gamma rays:

a) Some emitted photons have energies high enough to penetrate the scintillation crystal without being detected.

b) The number of emitted photons is typically low, which results in a noisy image

An image forming system (e.g. a colfimator) lowers the number of photons. However, if a knowledge of the spatial distribution of the sources is desired, one must have some gamma-flux loss due to collimation. Thus, one should choose a highly efficient detection system.

Bismuth germanate $\mathrm{Bi}_{4} \mathrm{Ge}_{3} \mathrm{O}_{12}$ (BGO) is a highly efficient scintillation detector, compared to the conventional $\mathrm{NaI}(\mathrm{Tl})$. This new scintillation material has approximately three times the stopping power of the traditional NaI(T1) $[1,2]$. This material may make it possible to detect high energy gamma-rays with higher efficiency than $\mathrm{NaI(Tl)}$ or with the same efficiency with a smaller size detector.

The purpose of this work was to investigate the possible usefulness of the BGO scintillator via Monte Carlo calculations for a slab geometry with various slab thicknesses over a wide energy range. These calculations determined the intrinsic efficiency, photon scattering within the crystal (single Compton as well as multiple scattering), the radial distribution of the energy deposition from the initial point of incidence, and the corresponding loss in image resolution and contrast. In these calculations no pulse height selection was assumed. This linearity of the scintillation response means that the distribution of energy deposition is proportional to the spatial distribution of light emitted by the scintillation crystal [3].

The method of calculation used here has been used by other authors [3]. Individual gamma ray histories for different energies are generated and stored for further analysis by a program which imposes a slab geometry. The study used the University of Michigan Amdahl 470V7 computer and generated 1000 incident gamma rays at the $(0,0,0)$ coordinates with the initial velocity vector oriented in the $Z$-direction.

The first step in the calculation was to sample the initial free path length using Monte Carlo techniques [3] with knowledge of the total cross section at that source energy. At the first interaction, the ratio of Compton to photoelectric cross sections was used to randomly select one of the two interaction processes with the aid of a random number generator subroutine [6] which produces by power residue method $2^{29}$ uniformly distributed random real numbers between 0 and 1.0 and random integers between 0 and $2^{31}$. If the initial event was photoelectric interaction then the history of the gamma photon was terminated and the photon assumed to be absorbed. If the initial interaction is a Compton scattering, then it was considered as a new photon of lower energy and new direction generated by randomly sampling the KleinNishina cross section using Kahn's [5] rejection method. The scattered photon was tracked further

\footnotetext{
* Present address: Nuclear Research Institute Tuwaitha, Baghdad, Iraq.
} 

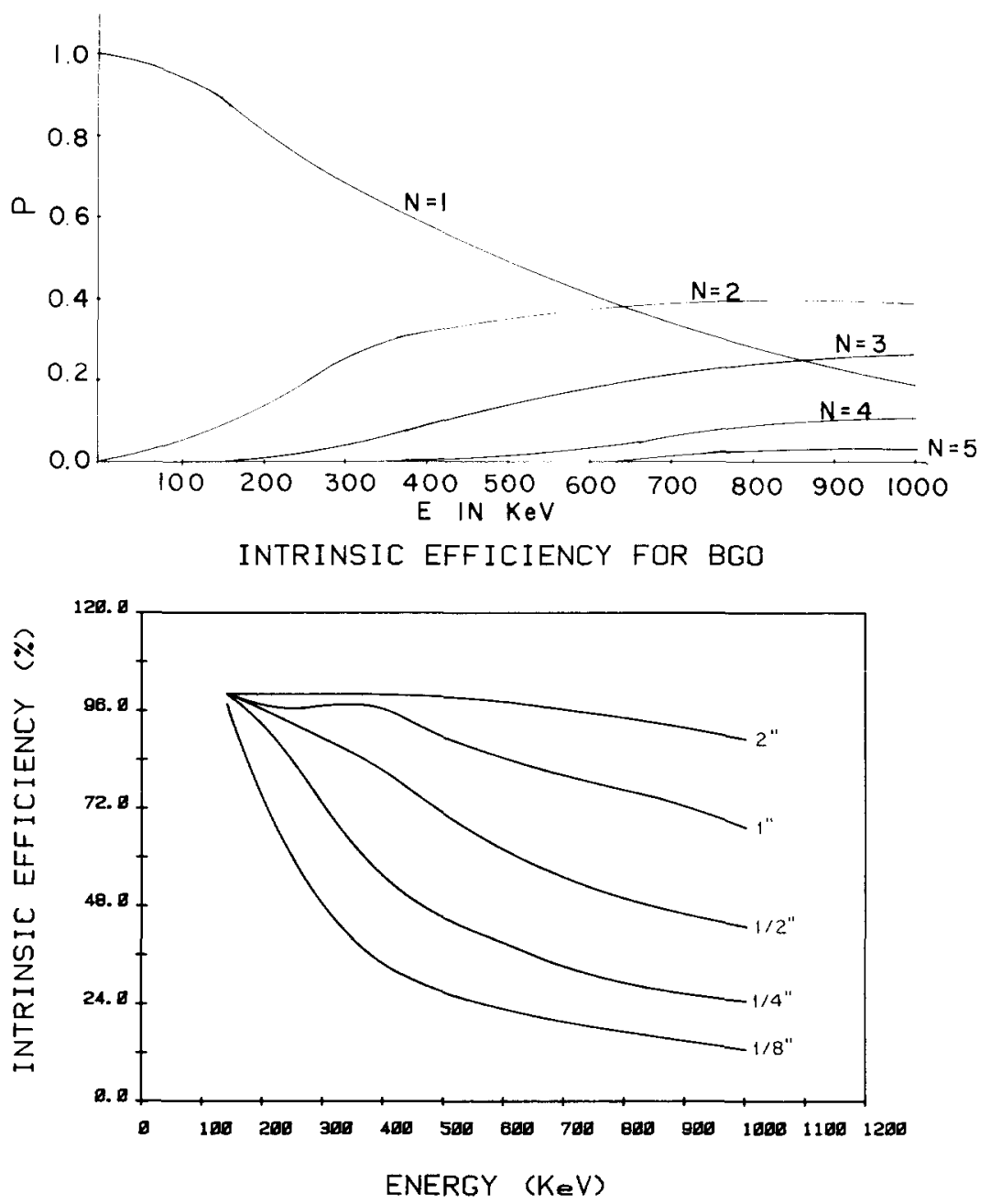

Fig. 1. Upper: probability of multiple interactions of gamma rays of energy $E$ in infinite medium of BGO. Lower: intrinsic efficiency for BGO of thickness range from $1 / 8^{\prime \prime}$ to $2^{\prime \prime}$.

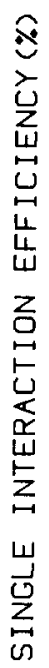

SINGLE INTERACTION EFFI. FOR BGO

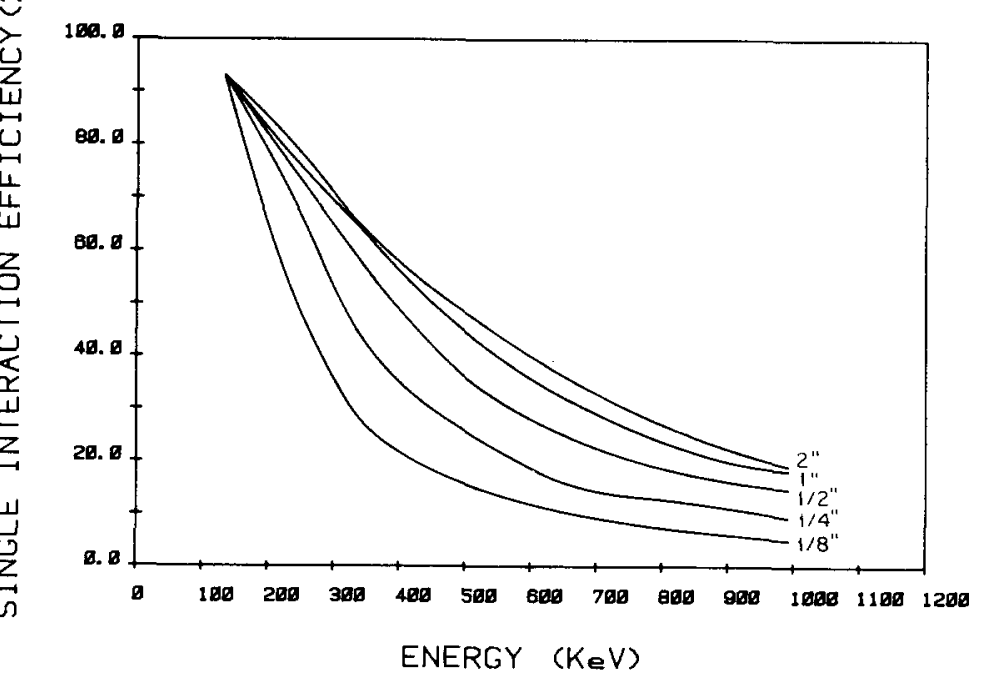

Fig. 2. Single interaction efficiency for BGO slab of thickness $1 / 8^{\prime \prime}$ to $2^{\prime \prime}$. 


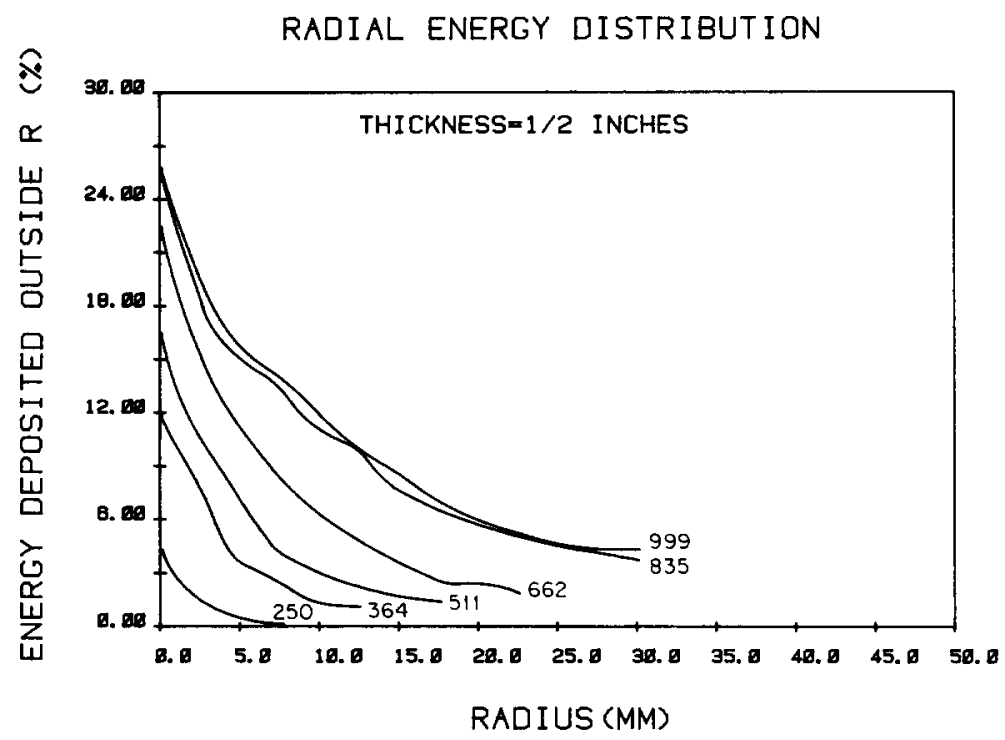

Fig. 3. Radial distribution of deposited energy for a $1 / 2$ " BGO slab for each vertically incident gamma ray of the indicated energy.

through the infinite medium until it was terminated by a photoelectron absorption.

In this program we used the cross sections compiled by Storm et al. [4]. Only photoelectric and Compton interactions were used in the energy range $140-1000 \mathrm{keV}$. The probabilities of a photon having $N$ interactions $(N-1$ scatterings and 1 photoabsorption) are plotted as functions of initial photon energy in fig. 1a. These clearly illustrate the increased probability of multiple scattering with increasing photon energy.

The second program used these infinite medium histories for each energy. It imposed a geometry of a slab of radius $4 \frac{1}{2}$ " and five thicknesses ranging from
$1 / 8^{\prime \prime}$ to $2^{\prime \prime}$. This program counts any history with any coordinates lying outside the disk as an escape photon. The program register the deposited energy and coordinates for each interaction that falls within the boundaries prior to an escape. Fig. 1b shows the variation of the intrinsic efficiency with detector thickness. Fig. 2 shows the relevant parameter to image formation: namely, the efficiency of the detector for photons that interact only once without degradation in their energy. This figure shows a weaker dependence on the thickness than that of the intrinsic efficiency shown in fig. 1b. Fig. 3 displays the radial distribution of deposited energy for BGO slab of thickness $1 / 2^{\prime \prime}$ for various gamma ray energies.

Table 1

Radial distribution of deposited energy for BGO slab as a function of thickness. $E=364 \mathrm{keV}$

\begin{tabular}{|c|c|c|c|c|c|}
\hline \multirow{2}{*}{$\begin{array}{l}\text { Percent energy deposited } \\
\text { outside radius } R \\
R(\mathrm{~mm})\end{array}$} & \multicolumn{5}{|c|}{ Thickness } \\
\hline & $1 / 8^{\prime \prime}$ & $1 / 4^{\prime \prime}$ & $1 / 2^{\prime \prime}$ & $1^{\prime \prime}$ & $2^{\prime \prime}$ \\
\hline 0 & 10.51 & 10.49 & 11.76 & 12.64 & 12.87 \\
\hline 2.5 & 7.59 & 7.22 & 7.65 & 8.29 & 8.41 \\
\hline 5.0 & 4.17 & 3.80 & 3.53 & 4.13 & 4.27 \\
\hline 7.5 & 2.86 & 2.77 & 2.38 & 2.66 & 2.84 \\
\hline 10.0 & 1.53 & 1.57 & 1.29 & 1.54 & 1.50 \\
\hline 12.5 & 1.29 & 1.31 & 1.10 & 1.30 & 1.25 \\
\hline 15.0 & 0.80 & 0.96 & 0.78 & 0.85 & 0.82 \\
\hline 17.5 & 0.53 & 0.63 & 0.54 & 0.56 & 0.54 \\
\hline 20.0 & 0.53 & 0.47 & 0.42 & 0.46 & 0.44 \\
\hline 22.5 & 0.26 & 0.16 & 0.11 & 0.19 & 0.18 \\
\hline
\end{tabular}




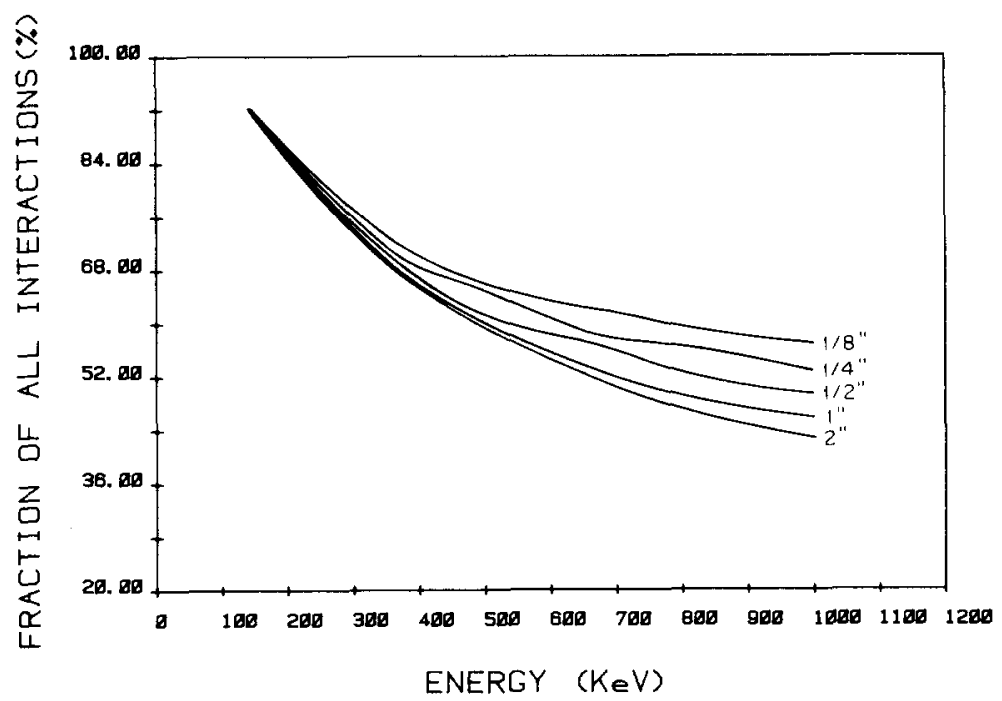

Fig. 4. The fraction of all types of interactions which occur at the point of incidence.

These calculations have been done for other thicknesses and showed only weak dependence on the thickness for each individual energy. Table 1 shows the radial distribution as a function of thickness. This means that one cannot improve the image much by increasing the thickness. This makes BGO attractive in the sense that even thin crystals give sharp images.
Fig. 4 shows another important parameter in image resolution, the fraction of all types of interactions which occur at the point of incidence. For two crystal thicknesses namely $1 / 4^{\prime \prime}$ and $1 / 2$ " we see shallow minima which means an improvement in image quality; this could be explained by having more forward scattering followed by escape around those

Table 2

Comparison of present results for BGO with those for NaI slabs [3] at $E=511 \mathrm{keV}$.

\begin{tabular}{|c|c|c|c|c|c|c|}
\hline Thickness & & $1 / 8^{\prime \prime}$ & $1 / 4^{\prime \prime}$ & $1 / 2^{\prime \prime}$ & $1^{\prime \prime}$ & $2^{\prime \prime}$ \\
\hline Intrinsic & BGO & 26.1 & 24.9 & 69.2 & 88.7 & 99.1 \\
\hline efficiency $(\%)$ & $\mathrm{Nal}$ & 10.2 & 19.9 & 35.4 & 57.8 & 82.6 \\
\hline $\begin{array}{l}\text { Probability of } \\
\text { history with }\end{array}$ & BGO & 13.3 & 22.2 & 32.9 & 41.8 & 46.4 \\
\hline $\begin{array}{l}\text { initial photo } \\
\text { interaction (\%) }\end{array}$ & $\mathrm{NaI}$ & 1.2 & 3.3 & 6.2 & 9.2 & 13.1 \\
\hline $\begin{array}{l}\text { Probability of } \\
\text { Compton (sin- }\end{array}$ & BGO & 76.8 & 59.7 & 34.4 & 13.8 & 2.6 \\
\hline $\begin{array}{l}\text { gle or multiple) } \\
\text { and escape }(\%)\end{array}$ & $\mathrm{NaI}$ & 7.1 & 12.3 & 17.6 & 21.8 & 18.0 \\
\hline Probability of & & & & & & \\
\hline $\begin{array}{l}\text { Compton (single } \\
\text { or multiple fol- }\end{array}$ & $\mathrm{BGO}$ & 9.9 & 18.1 & 32.9 & 44.6 & 51.2 \\
\hline $\begin{array}{l}\text { lowed by photo- } \\
\text { absorption }(\%)\end{array}$ & $\mathrm{NaI}$ & 1.9 & 4.2 & 11.7 & 26.8 & 51.5 \\
\hline $\begin{array}{l}\text { Probability of } \\
\text { full energy }\end{array}$ & BGO & 23.2 & 40.3 & 65.8 & 86.4 & 97.6 \\
\hline absorption (\%) & $\mathrm{NaI}$ & 3.1 & 7.6 & 17.9 & 36.0 & 64.6 \\
\hline
\end{tabular}


energies below these minima.

The results of these calculations show the BGO could be used up to about $660 \mathrm{keV}$ without serious deterioration in image resolution due to multiple interactions. It also indicates that smaller detectors than NaI(Tl) could be used for relatively high gamma energies $[1,2]$. Table 2 shows a comparison of various properties of BGO and $\mathrm{NaI}(\mathrm{Tl})$. These reflect some superiority of BGO over $\mathrm{NaI}(\mathrm{Tl})$ besides the advantages of durability and good performance without canning $[1,2]$.

The author would like to thank Prof. G.C. Summerfield for his careful reading of the manuscript.

\section{References}

[1] O.H. Nestor and Cy Huang, IEEE Trans. Nucl. Sci. NS-22 (1975) 68.

[2] Z.H. Cho and M.R. Farukhi, J. Nucl. Med. 18 (1977) 840.

[3] G.F. Knoll Nucl. Instr. and Meth. 71 (1969) 163;

H.O. Anger and D.H. Davis, Rev. Sci. Instr. 35 (1964) 693.

[4] E. Storm et al., LA-3753 Report, Photon cross sections from 0.001 to $100 \mathrm{MeV}$ for elements 1 through 100, Los Alamos, New Mexico (June 1967).

[5] H. Kahn, AECU-3259 (1954).

[6] IBM subroutine manual. 